\title{
Globular Clusters and Dark Satellite Galaxies Through the Stream Velocity
}

\section{Citation}

Naoz, Smadar, and Ramesh Narayan. 2014. "Globular Clusters and Dark Satellite Galaxies Through the Stream Velocity." The Astrophysical Journal 791 (1) (July 23): L8. doi:10.1088/2041-8205/791/1/18.

\section{Published Version}

doi:10.1088/2041-8205/791/1/L8

\section{Permanent link}

http://nrs.harvard.edu/urn-3:HUL.InstRepos:27802011

\section{Terms of Use}

This article was downloaded from Harvard University's DASH repository, and is made available under the terms and conditions applicable to Other Posted Material, as set forth at http:// nrs.harvard.edu/urn-3:HUL.InstRepos:dash.current.terms-of-use\#LAA

\section{Share Your Story}

The Harvard community has made this article openly available.

Please share how this access benefits you. Submit a story.

\section{Accessibility}




\title{
GLOBULAR CLUSTERS AND DARK SATELLITE GALAXIES THROUGH THE STREAM VELOCITY
}

\author{
SMAdAR NAOZ ${ }^{1,2,3}$ AND RAMESH NARAYAN ${ }^{1}$ \\ ${ }^{1}$ Institute for Theory and Computation, Harvard-Smithsonian Center for Astrophysics, 60 Garden Street, \\ Cambridge, MA 02138, USA; snaoz@astro.ucla.edu \\ ${ }^{2}$ Department of Physics and Astronomy, University of California, Los Angeles, CA 90095, USA \\ Received 2014 June 4; accepted 2014 July 11; published 2014 July 23
}

\begin{abstract}
The formation of purely baryonic globular clusters with no gravitationally bound dark matter is still a theoretical challenge. We show that these objects might form naturally whenever there is a relative stream velocity between baryons and dark matter. The stream velocity causes a phase shift between linear modes of baryonic and dark matter perturbations, which translates to a spatial offset between the two components when they collapse. For a $2 \sigma(3 \sigma)$ density fluctuation, baryonic clumps with masses in the range $10^{5}-2.5 \times 10^{6} M_{\odot}\left(10^{5}-4 \times 10^{6} M_{\odot}\right)$ collapse outside the virial radii of their counterpart dark matter halos. These objects could survive as long-lived, dark-matter-free objects and might conceivably become globular clusters. In addition, their dark matter counterparts, which were deprived of gas, might become dark satellite galaxies.
\end{abstract}

Key word: globular clusters: general

Online-only material: color figures

\section{INTRODUCTION}

Observations indicate that globular clusters (GCs) contain practically no gravitationally bound dark matter (DM; e.g., Heggie \& Hut 1996; Bradford et al. 2011; Conroy et al. 2011; Ibata et al. 2013). How did these objects form? Assuming a baryon-only universe, Peebles \& Dicke (1968) suggested in early work that GCs formed via gravitational collapse of nonlinear baryonic over-densities shortly after recombination $(z \sim 1000)$. Their model is no longer viable since we know now that DM dominates the matter content of the universe.

Gunn (1980) suggested that GCs are formed in strong shocks when gas is compressed during galaxy mergers. The discovery of many massive young star clusters in the interacting Antennae system (e.g., Whitmore \& Schweizer 1995; Whitmore et al. 1999) supports this idea, and the scenario has been incorporated in cosmological hierarchical structure formation models (e.g., Harris \& Pudritz 1994; Ashman \& Zepf 1992; Kravtsov \& Gnedin 2005; Muratov \& Gnedin 2010).

Another currently popular paradigm is that GCs, like all structure, initially formed inside DM halos (Peebles 1984), but these halos were later stripped by the tidal field of their host galaxies (e.g., Bromm \& Clarke 2002; Mashchenko \& Sills 2005; Saitoh et al. 2006; Bekki \& Yong 2012), leaving the central parts deficient in DM. However, some GCs are observed with stellar tidal tails, which is difficult to understand if the objects have extended DM halos (Grillmair et al. 1995; Moore 1996; Odenkirchen et al. 2003; Mashchenko \& Sills 2005).

In the standard model of structure formation, because of baryon-radiation coupling, baryon overdensities at the time of recombination were about five orders of magnitude smaller than DM over-densities. Baryons and DM also had different velocities at recombination, with a relative speed $\sim 30 \mathrm{~km} \mathrm{~s}^{-1}$ that was coherent on comoving scales of a few megaparsecs (Tseliakhovich \& Hirata 2010). After recombination, the baryons decoupled from the photons and their subsequent evo-

\footnotetext{
3 Einstein Fellow.
}

lution was dominated by the gravitational potential of the DM. They also cooled quickly, and their relative velocity with respect to the DM, called the "stream velocity," became supersonic.

The stream velocity has important implications for the first structures (Stacy et al. 2011; Maio et al. 2011; Greif et al. 2011; Fialkov et al. 2012; Naoz et al. 2012, 2013; O'Leary \& McQuinn 2012; Bovy \& Dvorkin 2012; Richardson et al. 2013; Tanaka \& Li 2014; Fialkov 2014), for the redshifted cosmological $21 \mathrm{~cm}$ signal (Dalal et al. 2010; Bittner \& Loeb 2011; Yoo et al. 2011; Visbal et al. 2012; McQuinn \& O'Leary 2012), and even for primordial magnetic fields (Naoz \& Narayan 2013).

Naoz et al. (2013) showed that the stream velocity can result in some halos becoming nearly baryon-free; the gas simply had too much relative velocity to fall into these DM halos. The question we ask here is: what happened to the baryons that failed to fall into these halos? We show that, in at least some cases, these baryons might have collapsed to form baryon-only bound objects that are physically separated from their parent DM halos. For an interesting range of masses, the spatial offset is larger than the virial radius of the DM halo, allowing the baryonic clumps to survive as independent DM-free objects. We suggest that these objects may have evolved into GCs. We also suggest that the corresponding gas-poor DM halos may be present-day dark satellites or ultra-faint galaxies.

We begin in Section 2 by discussing the evolution of baryonic overdensities in the presence of a stream velocity. We then calculate in Section 3 the likelihood of forming spatially separated baryon-only objects. We analyze the survival of these objects in Section 4 and conclude with a discussion in Section 5. Throughout, we adopt the following cosmological parameters: $\left(\Omega_{\Lambda}, \Omega_{m}, \Omega_{\mathrm{b}}, n, \sigma_{8}, H_{0}\right)=$ $\left(0.73,0.27,0.044,1.0,0.82,71 \mathrm{~km} \mathrm{~s}^{-1} \mathrm{Mpc}^{-1}\right)$.

\section{BARYONIC OVERDENSITY AND PHASE SHIFT}

We solve the coupled differential equations that govern the linear evolution of the dimensionless density fluctuations of the DM, $\delta_{\mathrm{dm}}$, and the baryons, $\delta_{\mathrm{b}}$. Both quantities are complex numbers. In the baryon frame of reference, the evolution 


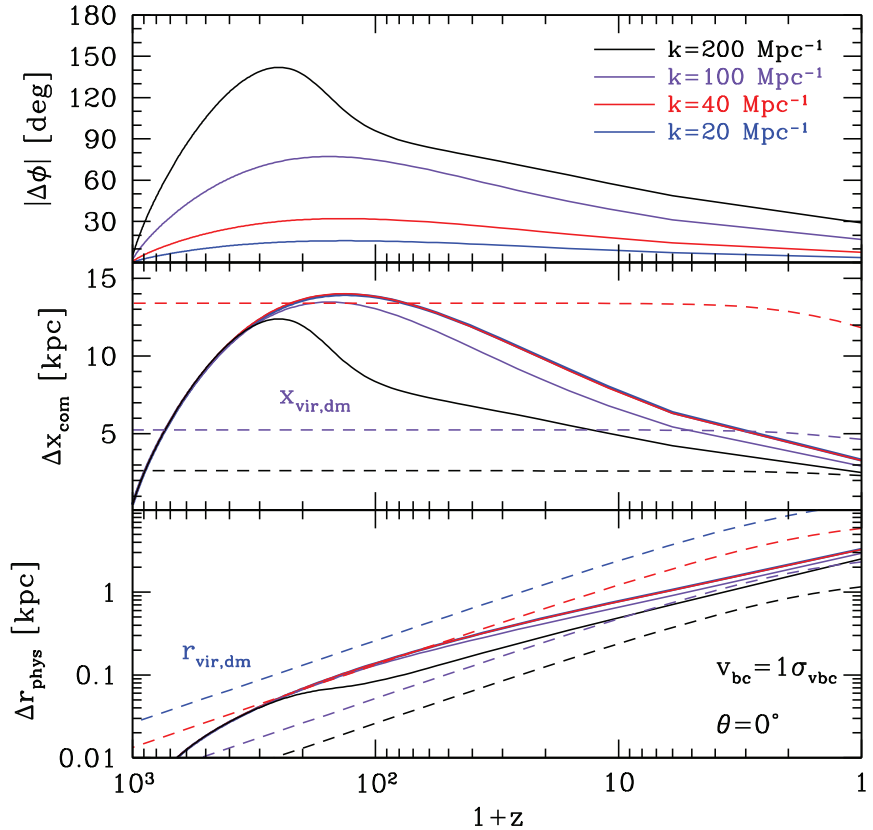

Figure 1. Top panel: phase shift $\Delta \phi$ between fluctuations in the baryons and $\mathrm{DM}$ as a function of redshift for modes with $k=200,100,40,20 \mathrm{Mpc}^{-1}$, assuming a stream velocity $v_{\mathrm{bc}}=1 \sigma_{\mathrm{vbc}}$ and $\theta=0$. Middle panel: comoving spatial separation between baryons and DM (solid lines) and comoving virial radius of the DM halo (dashed lines). Bottom panel: similar to the middle panel, but shows the physical spatial separation.

(A color version of this figure is available in the online journal.)

equations are

$$
\begin{aligned}
\ddot{\delta}_{\mathrm{dm}}+2 H \dot{\delta}_{\mathrm{dm}}-f_{\mathrm{dm}} \frac{2 i}{a} \mathbf{v}_{\mathrm{bc}} \cdot \mathbf{k} \dot{\delta}_{\mathrm{dm}}= & \frac{3}{2} H_{0}^{2} \frac{\Omega_{m}}{a^{3}}\left(f_{\mathrm{b}} \delta_{\mathrm{b}}+f_{\mathrm{dm}} \delta_{\mathrm{dm}}\right) \\
& +\left(\frac{\mathbf{v}_{\mathrm{bc}} \cdot \mathbf{k}}{a}\right)^{2} \delta_{\mathrm{dm}}
\end{aligned}
$$

$$
\ddot{\delta}_{\mathrm{b}}+2 H \dot{\delta}_{\mathrm{b}}=\frac{3}{2} H_{0}^{2} \frac{\Omega_{m}}{a^{3}}\left(f_{\mathrm{b}} \delta_{\mathrm{b}}+f_{\mathrm{dm}} \delta_{\mathrm{dm}}\right)-\frac{k^{2}}{a^{2}} \frac{k_{B} \bar{T}}{\mu}\left(\delta_{\mathrm{b}}+\delta_{T}\right),
$$

where $\Omega_{m}$ is the present-day matter density as a fraction of the critical density, $\mathbf{k}$ is the comoving wavenumber vector of the perturbation, $\mathbf{v}_{\mathrm{bc}}$ is the relative velocity between baryons and DM in a local patch of the universe, $a$ is the scale factor of the universe, $H_{0}$ is the present-day value of the Hubble parameter, $\mu$ is the mean molecular weight of the gas, $\bar{T}$ is the mean temperature of the baryons, $f_{\mathrm{b}}\left(f_{\mathrm{dm}}\right)$ is the cosmic baryon (DM) fraction, and $\delta_{T}$ is the dimensionless fluctuation in the baryon temperature. Derivatives are with respect to clock time. The above equations are a compact version of Equations (5) in Tseliakhovich \& Hirata (2010). We have used the fact that $v_{\mathrm{bc}} \propto 1 / a$, and have included a pressure term appropriate to the equation of state of an ideal gas (Naoz \& Barkana 2005).

The density perturbation amplitudes $\delta_{\mathrm{b}}$ and $\delta_{\mathrm{dm}}$ are complex numbers, with phases given by

$$
\phi_{\mathrm{b}, \mathrm{dm}}=\arctan \left[\operatorname{Im}\left(\delta_{\mathrm{b}, \mathrm{dm}}\right) / \operatorname{Re}\left(\delta_{\mathrm{b}, \mathrm{dm}}\right)\right] \text {. }
$$

The stream velocity introduces a phase shift, $\Delta \phi=\phi_{\mathrm{b}}-\phi_{\mathrm{dm}}$, between the baryons and DM (because of the third term in the left-hand side of Equation (1)). This phase shift translates to a physical separation between the DM and baryon overdensities.

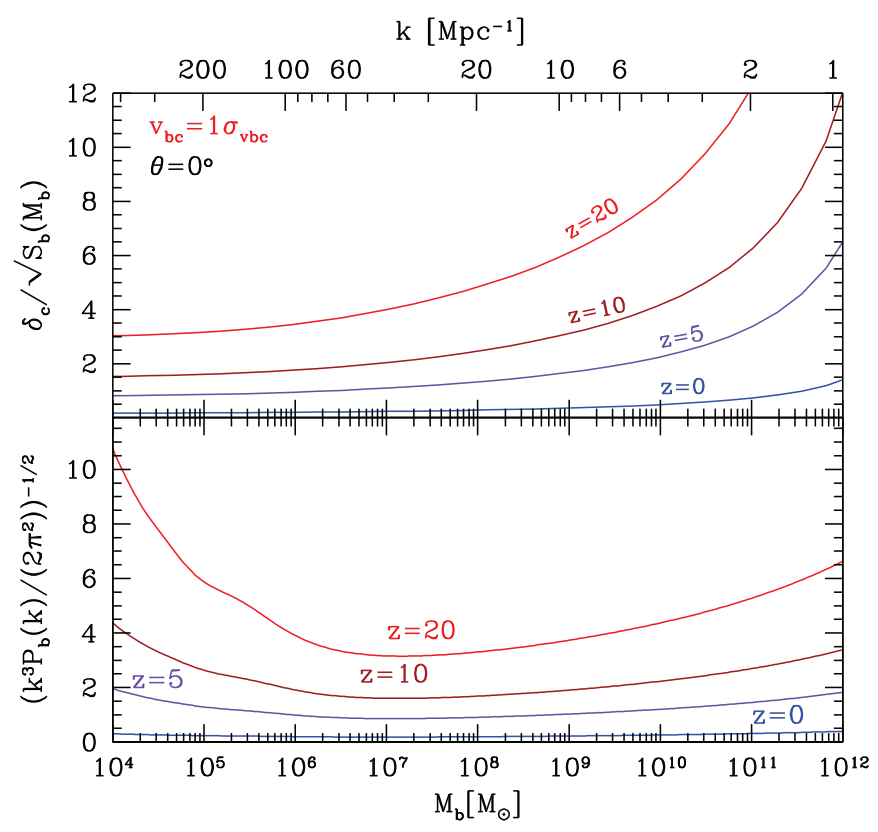

Figure 2. Top panel: the vertical axis measures the rarity of fluctuations ("number of $\sigma$ 's") needed to produce a baryonic clump of mass $M_{\mathrm{b}}$ (horizontal axis) at a given redshift (different solid lines); $\delta_{c}=1.686$ is the critical overdensity for collapse and $S_{\mathrm{b}}$ is the variance of $\delta_{\mathrm{b}}$ (Equation (6)). Results are for a stream velocity $v_{\mathrm{bc}}=1 \sigma_{\mathrm{vbc}}$ and $\theta=0$. Bottom panel: corresponding results for a different measure of fluctuation rarity, $1 /\left|\delta_{\mathrm{b}}\right|=1 / \sqrt{k^{3} P_{\mathrm{b}}(k) /\left(2 \pi^{2}\right)}$.

(A color version of this figure is available in the online journal.)

The phase shift was discussed previously by Naoz et al. (2012), but the corresponding spatial shift was not resolved in their simulations. Here we use analytical linear theory.

Since the phase shift depends on $\mathbf{v}_{\mathrm{bc}} \cdot \mathbf{k}$, both the angle $\theta$ between $\mathbf{v}_{\mathrm{bc}}$ and $\mathbf{k}$ and the magnitude of $v_{\mathrm{bc}}$ are relevant. For concreteness, we present results corresponding to $\theta=0$ and $v_{\mathrm{bc}}=1 \sigma_{\mathrm{vbc}}$, where $\sigma_{\mathrm{vbc}}$ is the (scale-independent) rms fluctuation of the stream velocity on small scales. In the top panel of Figure 1, we show $\Delta \phi$ as a function of redshift for four representative wavenumbers, $k=200,100,40$, and $20 \mathrm{Mpc}^{-1}$, which correspond to baryon masses $M_{\mathrm{b}} \sim 10^{5}, 10^{6}, 10^{7}, 10^{8} M_{\odot}$, respectively (Figure 2). As seen in Figure 1, smaller scales (larger wavenumbers) develop a larger $\Delta \phi$. The phase difference is related to the comoving distance between the baryon and DM fluctuation peaks, $\Delta x_{\text {com }}$, by

$$
\Delta x_{\mathrm{com}}=\left(\Delta \phi / 360^{\circ}\right)(2 \pi / k) .
$$

This comoving separation $\Delta x_{\text {com }}$, as well as the corresponding physical separation, $\Delta r_{\text {phys }}=\Delta x_{\text {com }} /(1+z)$, are shown in the lower two panels of Figure 1.

To evaluate how significant the spatial displacement between the baryon and DM overdensities is, we compare it to the virial radius of the DM-only nonlinear object. The virial radius of an object that collapses at redshift $z$ is approximately (Bryan \& Norman 1998)

$$
r_{\mathrm{vir}, \mathrm{dm}} \approx 0.784\left(\frac{M_{\mathrm{dm}}}{10^{8} M_{\odot} h^{-1}}\right)^{1 / 3}\left(\frac{1+z}{10}\right)^{-1} h^{-1} \mathrm{kpc},
$$

where, for simplicity, we have suppressed a weak dependence on the cosmological constant, which causes a slight decrease of the virial radius at low redshift (this effect is included in the numerical calculations). The comoving virial radius is given by $x_{\mathrm{vir}, \mathrm{dm}}=r_{\mathrm{vir}, \mathrm{dm}}(1+z)$. 
Over a substantial range of $z$, modes with $k=$ 200, $100 \mathrm{Mpc}^{-1}$ (or $M_{\mathrm{b}} \sim 10^{5}, 10^{6} M_{\odot}$ ) have spatial offsets between their baryonic and DM linear overdensity peaks larger than the DM virial radius (Figure 1). If baryons are able to collapse at these redshifts, they will form isolated baryonic clumps. In contrast, modes with smaller values of $k$ (larger $\left.M_{\mathrm{b}}\right)$ have separations that lie within the DM virial radius.

\section{LIKELIHOOD OF BARYON-ONLY CLUMP FORMATION}

When the baryon overdensity amplitude $\left|\delta_{\mathrm{b}}\right|$ approaches unity, the perturbation becomes nonlinear and we expect the baryons to collapse. To estimate how rare such collapsed objects are we calculate the variance of $\delta_{\mathrm{b}}$ as a function of baryon clump mass $M_{\mathrm{b}}$ :

$$
\begin{aligned}
S_{\mathrm{b}}\left(M_{\mathrm{b}}, z\right) & =\left\langle\left|\delta_{\mathrm{b}}\left(M_{\mathrm{b}}, z\right)\right|^{2}\right\rangle \\
& =\int_{0}^{\infty} \frac{d k}{2 \pi^{2}} k^{2} P_{\mathrm{b}}(k)\left[\frac{3 j_{1}(k R)}{k R}\right]^{2},
\end{aligned}
$$

where $P_{\mathrm{b}}(k)$ is the spectrum of baryon fluctuations calculated using Equations (1)-(3), $j_{1}(x)=(\sin x-x \cos x) / x^{2}$, and the scale $R$ is the radius of a top-hat window function, with $M_{\mathrm{b}}=4 \pi \rho_{\mathrm{b}} R^{3} / 3$. The value of $S_{\mathrm{b}}\left(M_{\mathrm{b}}, z\right)$ is normalized to $\sigma_{8 \text {,tot }}(z=0)$ of the total matter. If $\delta_{c}$ is the critical linear overdensity for collapse, the rarity of clumps of mass $M_{\mathrm{b}}$ at redshift is determined by the number of $\sigma$ fluctuations needed: $\delta_{c} / \sqrt{S_{\mathrm{b}}\left(M_{\mathrm{b}}, z\right)}$ (e.g., Barkana \& Loeb 2001; Naoz \& Barkana 2007). For simplicity, we have set $\delta_{c}$ to its standard value of 1.686 (but note that $\delta_{c}$ may vary with time; Naoz et al. 2006; Fialkov et al. 2012).

We estimate the mass $M_{\mathrm{b}}$ of a baryon-only clump that forms from a mode with comoving wavenumber $k$ by

$$
M_{\mathrm{b}}=\frac{4 \pi}{3} \bar{\rho}_{\mathrm{b}, 0}\left(\frac{1}{2} \frac{2 \pi}{k}\right)^{3}=\frac{1}{2} \frac{H_{0}^{2} \Omega_{\mathrm{b}}}{G}\left(\frac{1}{2} \frac{2 \pi}{k}\right)^{3} .
$$

In the top panel of Figure 2, we show the number of $\sigma$ 's by which an overdensity of a given baryon mass $M_{\mathrm{b}}$ must fluctuate in order for it to collapse at a given redshift. As an example, a $2 \sigma$ baryon fluctuation with a mass $M_{\mathrm{b}}=10^{6} M_{\odot}$ collapses at $z=11.4$.

The quantity $\delta_{c} / \sqrt{S_{\mathrm{b}}\left(M_{\mathrm{b}}, z\right)}$ is a little misleading since $S_{\mathrm{b}}$ is computed as an integral over wavenumber $k$. While the window function cuts off the contribution of all $k$ larger than about $1 / R$, there is no cutoff at low $k$ (large masses). Thus, $S_{\mathrm{b}}\left(M_{\mathrm{b}}\right)$ has a contribution from fluctuations with masses much larger than $M_{\mathrm{b}}$. This contamination is usually not important, but it is serious when $M_{\mathrm{b}}$ lies below the Jeans scale so that the collapse of baryon overdensities is suppressed by gas pressure. In this situation, $\delta_{c} / \sqrt{S_{\mathrm{b}}\left(M_{\mathrm{b}}, z\right)}$ may indicate collapse at the $M_{\mathrm{b}}$ of interest even though what is collapsing is actually some larger mass that is unaffected by gas pressure. To illustrate this point, we show in the bottom panel of Figure 2 a different measure of the rarity of fluctuations, $1 /\left|\delta_{\mathrm{b}}\right|=1 / \sqrt{k^{3} P_{\mathrm{b}}(k) /\left(2 \pi^{2}\right)}$, which focuses on the local power in baryon density fluctuations at wavenumber $k$. Note the clear signature of the Jeans cutoff at low masses and high redshifts.

It is important to note that the collapse of baryonic clumps considered here is very different from collapse in the baryononly universe considered by Peebles \& Dicke (1968). In our case, baryons feel the gravitational potential of the DM; their collapse is driven primarily by the DM. However, because of the stream velocity, by the time the baryons collapse, they are spatially offset from the corresponding DM halo.

\section{SURVIVAL OF BARYONIC CLUMPS}

When a baryonic perturbation collapses, the spatial separation of the baryon clump from its DM halo is $\Delta r_{\text {phys }}=\Delta x_{\text {com }} /(1+z)$, where $\Delta x_{\text {com }}$ is obtained by evaluating Equation (4) at the redshift of collapse. The bottom panel in Figure 1 shows $\Delta r_{\text {phys }}$ for some cases of interest. The upper panels in Figure 3 show the same information in a different format. For each $k$, or equivalently each baryon clump mass $M_{\mathrm{b}}$, we have identified from Figure 2 the redshift at which a $2 \sigma(3 \sigma)$ fluctuation collapses, ${ }^{4}$ and computed the ratio of $\Delta r_{\text {phys }}$ at this $z$ to the virial radius $r_{\mathrm{vir}, \mathrm{dm}}$ (Equation (5)) of the just-collapsed DM halo. For example, a $2 \sigma$ fluctuation with $M_{\mathrm{b}} \approx 2.5 \times 10^{6} M_{\odot}$ collapses with a spatial offset exactly equal to the virial radius of its DM halo. Clumps with smaller mass (larger wavenumber) form outside the virial radius, and vice versa. In the case of a $3 \sigma$ fluctuation, the transition mass is $M_{\mathrm{b}} \approx 4 \times 10^{6} M_{\odot}$.

Baryon clumps that form inside the virial radius of the DM host will spiral down to the center by dynamical friction. To estimate the timescale we adopt the fitting formula presented in Boylan-Kolchin et al. (2008):

$$
t_{\mathrm{df}, \mathrm{BK}}=0.22 \frac{\left(M_{\mathrm{dm}} / M_{\mathrm{b}}\right)^{1.3}}{\ln \left(1+M_{\mathrm{dm}} / M_{\mathrm{b}}\right)} \frac{\Delta r_{\mathrm{phys}}}{r_{\mathrm{vir}, \mathrm{dm}}} e^{1.9 \sqrt{1-e_{\mathrm{b}}^{2}}}\left(\frac{r_{\mathrm{vir}, \mathrm{dm}}^{3}}{G M_{\mathrm{dm}}}\right)^{1 / 2},
$$

where $e_{\mathrm{b}}$ is the eccentricity of the baryon clump's orbit, which we set to 0.5 . We add $t_{\mathrm{df}, \mathrm{BK}}$ to the epoch of collapse $t_{\text {coll }}$ and show the sum in Figure 3 (lower panels).

Baryon clumps that form outside the virial radius are not affected by dynamical friction. However, these clumps generally have lower masses and are liable to lose any stars that they form via evaporation. Following Gnedin et al. (2014) and Gieles et al. (2011), we estimate the evaporation timescale as

$$
t_{\text {evap }}=17 \mathrm{Gyr} \frac{M_{\mathrm{b}}}{2 \times 10^{5} M_{\odot}} .
$$

Again, we plot the sum $t_{\text {evap }}+t_{\text {coll }}$.

Figure 3 shows that baryon clumps with $M_{\mathrm{b}} \sim 10^{5}-4 \times$ $10^{6} M_{\odot}$ may be able to survive destruction by either dynamical friction or evaporation, and may survive as independent longlived clumps. These may be the objects we see today as GCs. Furthermore, their parent DM halos, which collapsed with a deficit of baryons, may today be ultra-faint galaxies and dark satellite galaxies.

While the above proposal seems attractive, could baryon clumps in the favorable mass range $M_{\mathrm{b}} \sim 10^{5}-4 \times 10^{6} M_{\odot}$ fall into their parent DM halos simply through the gravitational pull of the latter. The free-fall time from a separation $\Delta r_{\text {phys }}$ is very short:

$$
\begin{aligned}
t_{\mathrm{ff}} & =\pi \frac{\left(\Delta r_{\mathrm{phys}} / 2\right)^{3 / 2}}{\sqrt{G\left(M_{\mathrm{b}}+M_{\mathrm{dm}}\right)}} \\
& =0.27 \mathrm{Gyr}\left(\frac{\Delta r_{\mathrm{phys}}}{0.59 \mathrm{kpc}}\right)^{3 / 2}\left(\frac{\Omega_{m}}{\Omega_{b}} \frac{M_{\mathrm{b}}}{10^{6} M_{\odot}}\right)^{-1 / 2} .
\end{aligned}
$$

\footnotetext{
4 Since GCs account for only a small fraction of the baryon content of the universe, they are clearly rare, hence we focus on $2 \sigma$ and $3 \sigma$ fluctuations, rather than the more common $1 \sigma$ fluctuations.
} 


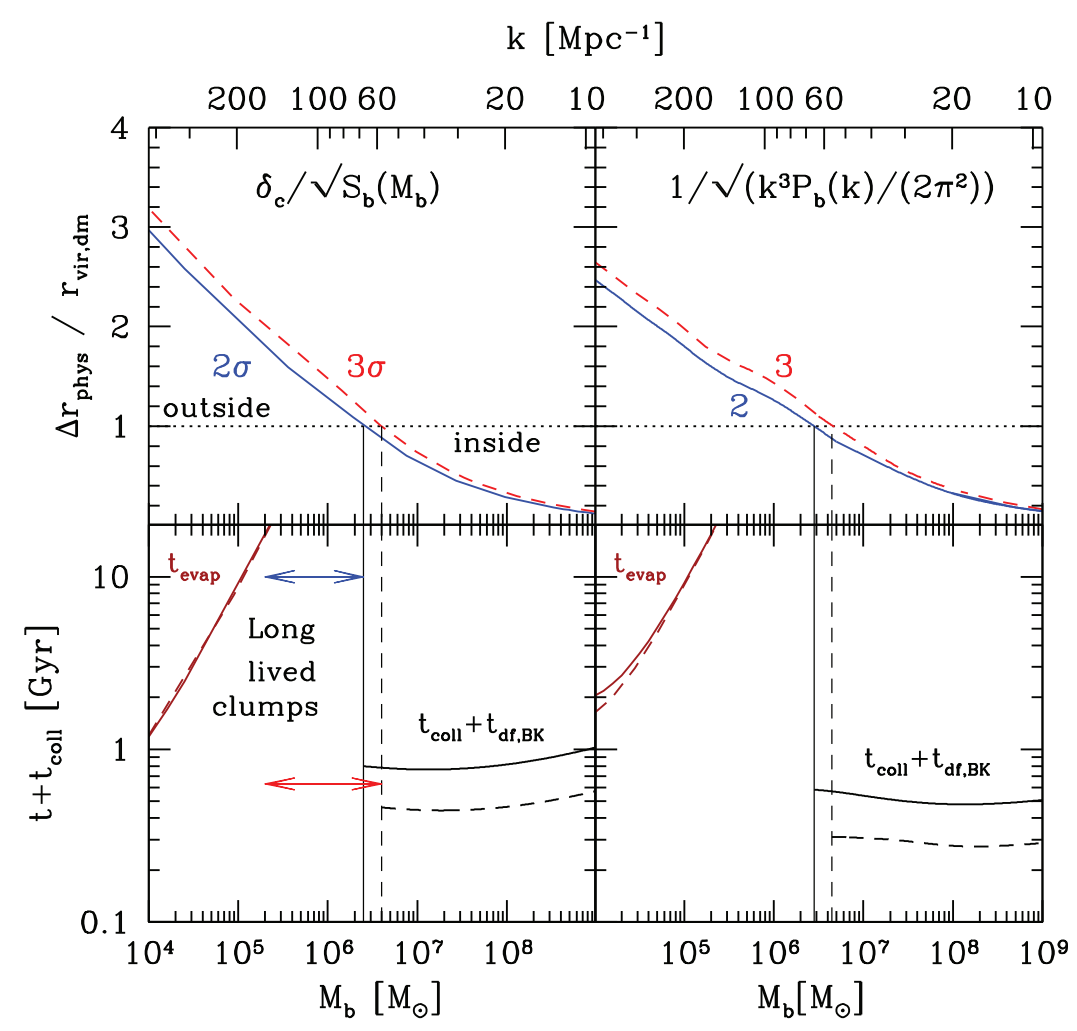

Figure 3. Top left panel: spatial separation between baryon and DM clumps at the redshift of collapse, normalized by the virial radius of the DM halo, for $2 \sigma$ (solid line) and $3 \sigma$ (dashed line) fluctuations, plotted against the baryon clump mass $M_{\mathrm{b}}$. Collapse is defined by the condition $\delta_{c} / \sqrt{S_{\mathrm{b}}\left(M_{\mathrm{b}}\right)}=2,3$, for $2 \sigma$ and $3 \sigma$. Note that clumps with baryon mass larger than a few $\times 10^{6} M_{\odot}$ are separated from their DM halos by less than the virial radius (they are "inside" the DM halo) and vice versa for clumps with smaller masses ("outside"). Calculations are for $v_{\mathrm{bc}}=1 \sigma_{\mathrm{vbc}}, \theta=0$. Bottom left panel: estimated survival time of baryon clumps against spiral-in and merger through dynamical friction ("inside" the DM halo) and loss of stars through evaporation ("outside" the halo). Baryon clumps with masses in the range $\sim 10^{5}-4 \times 10^{6} M_{\odot}$ are potentially long-lived, especially if tidal forces from other nearby clumps unbind them from their DM halos. Right panels: similar to left panels, except that collapse is defined by $1 / \sqrt{k^{3} P_{\mathrm{b}}(k) /\left(2 \pi^{2}\right)}=2,3$. The results are generally similar.

(A color version of this figure is available in the online journal.)

(Note a $2 \sigma$ fluctuation of $M_{\mathrm{b}}=10^{6} M_{\odot}$ has a physical separation of $0.59 \mathrm{kpc}$ from its DM host.) The actual timescale is a little longer since the baryon clump will begin with an outward velocity (Hubble flow). However, this changes the result by less than a factor of two.

Newly formed baryon and DM clumps will certainly free-fall and merge if they evolve in isolation. More often, however, we expect the two objects to participate in the hierarchical growth of structure in the universe. At least some baryon clumps that collapse outside the virial radius of their DM parent halos will experience strong tidal forces from neighboring objects and will become gravitationally unbound from their DM halos. It remains to be seen if a sufficient number can survive by this mechanism to explain the GCs we observe in the current universe. ${ }^{5}$

\section{DISCUSSION}

We have used linear theory to study the growth of baryonic and DM density fluctuations in the universe in the presence of a stream velocity $v_{\mathrm{bc}}$ between the two components. We focused on the fact that a non-zero stream velocity causes a phase shift $\Delta \phi$ between the complex amplitudes of the baryonic and DM density fluctuations, which results in a spatial separation

\footnotetext{
5 Note that a previous study by O'Leary \& McQuinn (2012) focused on $1 \sigma$ fluctuations, which collapse at redshifts well below 10 (according to our analysis), whereas their numerical simulation was limited to $z>10$. Furthermore, because of low statistical sampling, they were unable to follow the evolution of $2 \sigma$ and $3 \sigma$ fluctuations. This may explain why they did not see any baryon-only clumps such as we predict.
}

between the two density peaks. When the perturbations go nonlinear and collapse, the baryon clump forms at a different spatial location than its DM counterpart. For baryon clump masses less than about few $\times 10^{6} M_{\odot}$, the separation is larger than the virial radius of the DM halo. Assuming tidal forces from other nearby objects are able to unbind the baryon clump from its DM halo, the clump could survive to the present day as a DMfree gravitationally self-bound object. We suggest that this may be how GCs formed in the universe. The corresponding baryondeficient DM halos would similarly survive as dark satellite galaxies or ultra-faint galaxies, as suggested previously by $\mathrm{NaOZ}$ et al. (2013).

Note that in this picture, the collapse of a baryon clump is not driven purely by the self-gravity of the baryons. The primary driving agent is still the DM perturbation, whose effect on the baryons is nearly the same as in the standard $\left(v_{\mathrm{bc}}=0\right)$ model of structure formation, so long as the phase shift $\Delta \phi$ is less than about a radian. For perturbations that satisfy this condition, baryonic collapse is almost as effective as in the standard model, and gravity is able to overcome gas pressure; the only difference is that the baryon and DM clumps form in spatially distinct locations.

In Figure 1 we showed phase and spatial offsets between the baryon and DM perturbations for linear modes with different wavenumbers $k$. As an example, at $z \approx 11.4$, a mode with $k \approx 100 \mathrm{Mpc}^{-1}$ (which corresponds to a baryonic mass $M_{\mathrm{b}}=10^{6} M_{\odot}$ ), has a phase shift $\sim 39^{\circ}$, which translates to a comoving distance between the baryon and DM density peaks of $7.3 \mathrm{kpc}$. A $2 \sigma$ fluctuation with this $k$ collapses at $z \approx 11.4$ 
(Figure 2), and the spatial offset between the baryonic and DM clumps is about 1.3 times the virial radius of the DM halo. For a $3 \sigma$ fluctuation, collapse occurs at an earlier redshift and the separation is even larger.

Using the virial radius of the DM halo as a benchmark spatial separation to discriminate between baryon clumps that survive and those that merge through dynamical friction, we obtain a natural upper cutoff to the mass of baryon-only clumps of a few $\times 10^{6} M_{\odot}$ (Figure 3 ). There is similarly a natural lower cutoff at around $10^{5} M_{\odot}$, which arises from a combination of several effects: survival against evaporation of stars (Figure 3), Jeans cutoff due to gas pressure, and too large a phase shift between baryons and DM (which is a serious effect for $k>$ a few hundred $\mathrm{Mpc}^{-1}$ ). The resulting mass range of long-lived clumps, $M_{\mathrm{b}} \sim 10^{5}-$ few $\times 10^{6} M_{\odot}$, agrees well with the masses of present-day GCs. ${ }^{6}$ Note that all the numerical estimates given in this Letter are for $v_{\mathrm{bc}}=1 \sigma_{\mathrm{vbc}}$ and $\theta=0$. The range extends to larger masses for $v_{\mathrm{bc}}=2 \sigma_{\mathrm{vbc}} ; \theta=\pi$ is equivalent to $\theta=0$, but $\theta=\pi / 2$ has no stream velocity effect (within linear theory).

A great deal of work is needed before one can be confident that the baryon-only clumps discussed here can indeed form naturally and that they are sufficiently long-lived to be interesting. Within an analytical approach, one has to consider higher-order nonlinear techniques. Alternatively, a numerical approach is possible, but it will require much better mass resolution than one usually finds in galaxy formation simulations. Assuming one successfully demonstrates that the baryon clumps proposed here form and are long-lived, one must test whether or not stars will form inside the collapsed clumps, and whether a sufficient number of stars will survive down to $z=0$ with the correct spatial and metallicity distribution. These are challenging problems.

Finally we note that, even for baryonic (and DM) masses much larger than those discussed here (arising from modes with $k \ll 10 \mathrm{Mpc}^{-1}$ ), there is a significant spatial offset between the baryons and DM at the time of collapse ( $\sim 1-3 \mathrm{kpc}$, depending on the redshift; Figure 1). These baryon and DM clumps will quickly merge through dynamical friction. However, in the process, the baryons will likely cause substantial stirring of the DM fluid in the inner regions of the halo. This might well produce a core-like structure in the DM density distribution, potentially explaining a number of puzzling observations (for a review, see de Blok 2010). Also, as Figure 1 indicates, the spatial offset $\Delta r_{\text {phys }}$ evolves significantly with the redshift of formation. This means that galaxies that formed at higher redshifts are likely to have smaller DM cores, and their baryonic components will also be more compact, compared to galaxies that formed more recently (e.g., Daddi et al. 2005; van Dokkum et al. 2008). These topics are beyond the scope of this Letter.

We thank Avi Loeb, Rennan Barkana, Steve Furlanetto, Mark Vogelsberger, and the anonymous referee for useful comments.
S.N. was supported by NASA through an Einstein Post-doctoral Fellowship awarded by the Chandra X-ray Center, operated by the Smithsonian Astrophysical Observatory for NASA, contract PF2-130096. R.N. was supported in part by NSF grant AST1312651.

\section{REFERENCES}

Ashman, K. M., \& Zepf, S. E. 1992, ApJ, 384, 50

Barkana, R., \& Loeb, A. 2001, PhR, 349, 125

Bekki, K., \& Yong, D. 2012, MNRAS, 419, 2063

Bittner, J. M., \& Loeb, A. 2011, arXiv:1110.4659

Bovy, J., \& Dvorkin, C. 2012, ApJ, 768, 70

Boylan-Kolchin, M., Ma, C.-P., \& Quataert, E. 2008, MNRAS, 383, 93

Bradford, J. D., Geha, M., Muñoz, R. R., et al. 2011, ApJ, 743, 167

Bromm, V., \& Clarke, C. J. 2002, ApJL, 566, L1

Bryan, G. L., \& Norman, M. L. 1998, ApJ, 495, 80

Conroy, C., Loeb, A., \& Spergel, D. N. 2011, ApJ, 741, 72

Daddi, E., Renzini, A., Pirzkal, N., et al. 2005, ApJ, 626, 680

Dalal, N., Pen, U., \& Seljak, U. 2010, JCAP, 11, 007

de Blok, W. J. G. 2010, AdAst, 2010, 789293

Fialkov, A. 2014, arXiv:1407.2274

Fialkov, A., Barkana, R., Tseliakhovich, D., \& Hirata, C. M. 2012, MNRAS, 424, 1335

Gieles, M., Heggie, D. C., \& Zhao, H. 2011, MNRAS, 413, 2509

Gnedin, O. Y., Ostriker, J. P., \& Tremaine, S. 2014, ApJ, 785, 71

Greif, T., White, S., Klessen, R., \& Springel, V. 2011, ApJ, 736, 147

Grillmair, C. J., Freeman, K. C., Irwin, M., \& Quinn, P. J. 1995, AJ, 109, 2553

Gunn, J. E. 1980, in Globular Clusters, ed. D. Hanes \& B. Madore (Cambridge: Cambridge Univ. Press), 301

Harris, W. E., \& Pudritz, R. E. 1994, ApJ, 429, 177

Heggie, D. C., \& Hut, P. 1996, in IAU Symp. 174, Dynamical Evolution of Star Clusters: Confrontation of Theory and Observations, ed. P. Hut \& J. Makino (Dordrecht: Kluwer), 303

Ibata, R., Nipoti, C., Sollima, A., et al. 2013, MNRAS, 428, 3648

Kravtsov, A. V., \& Gnedin, O. Y. 2005, ApJ, 623, 650

Maio, U., Koopmans, L. V. E., \& Ciardi, B. 2011, MNRAS, 412, L40

Mashchenko, S., \& Sills, A. 2005, ApJ, 619, 258

McQuinn, M., \& O'Leary, R. M. 2012, ApJ, 760, 3

Moore, B. 1996, ApJL, 461, L13

Muratov, A. L., \& Gnedin, O. Y. 2010, ApJ, 718, 1266

Naoz, S., \& Barkana, R. 2005, MNRAS, 362, 1047

Naoz, S., \& Barkana, R. 2007, MNRAS, 377, 667

Naoz, S., \& Narayan, R. 2013, PhRvL, 111, 051303

Naoz, S., Noter, S., \& Barkana, R. 2006, MNRAS, 373, L98

Naoz, S., Yoshida, N., \& Gnedin, N. Y. 2012, ApJ, 747, 128

Naoz, S., Yoshida, N., \& Gnedin, N. Y. 2013, ApJ, 763, 27

Odenkirchen, M., Grebel, E. K., Dehnen, W., et al. 2003, AJ, 126, 2385

O'Leary, R. M., \& McQuinn, M. 2012, ApJ, 760, 4

Peebles, P. J. E. 1984, ApJ, 277, 470

Peebles, P. J. E., \& Dicke, R. H. 1968, ApJ, 154, 891

Richardson, M. L. A., Scannapieco, E., \& Thacker, R. J. 2013, ApJ, 771, 81

Saitoh, T. R., Koda, J., Okamoto, T., Wada, K., \& Habe, A. 2006, ApJ, 640,22

Stacy, A., Bromm, V., \& Loeb, A. 2011, ApJL, 730, L1

Tanaka, T. L., \& Li, M. 2014, MNRAS, 439, 1092

Tseliakhovich, D., \& Hirata, C. 2010, PhRvD, 82, 083520

van Dokkum, P. G., Franx, M., Kriek, M., et al. 2008, ApJL, 677, L5

Visbal, E., Barkana, R., Fialkov, A., Tseliakhovich, D., \& Hirata, C. M. 2012, Natur, 487, 70

Whitmore, B. C., \& Schweizer, F. 1995, AJ, 109, 960

Whitmore, B. C., Zhang, Q., Leitherer, C., et al. 1999, AJ, 118, 1551

Yoo, J., Dalal, N., \& Seljak, U. 2011, JCAP, 07, 018

\footnotetext{
6 Even if star formation had a relatively low efficiency in these pristine gas clumps (say $\sim 10 \%$ ), a $4 \times 10^{6} M_{\odot}$ baryon clump would still have produced enough stars to make it an attractive candidate progenitor for present-day GCs. The topic of star formation in our baryon-only clumps is beyond the scope of this Letter.
} 